\title{
Randomized controlled trial of a special acupuncture technique for pain after thoracotomy
}

\author{
Gary Deng, MD, PhD, ${ }^{\text {a }}$ Valerie Rusch, MD, ${ }^{\mathrm{b}}$ Andrew Vickers, PhD, ${ }^{\mathrm{a}, \mathrm{c}}$ Vivek Malhotra, MD, ${ }^{\mathrm{d}}$ Pamela Ginex, RN, \\ Robert Downey, MD, ${ }^{\mathrm{b}}$ Manjit Bains, MD, ${ }^{\mathrm{b}}$ Bernard Park, MD, ${ }^{\mathrm{b}}$ Nabil Rizk, MD, ${ }^{\mathrm{b}}$ Raja Flores, MD, ${ }^{\mathrm{b}}$ Simon Yeung, RPh, \\ and Barrie Cassiletha, $\mathrm{PhD}^{\mathrm{a}}$
}

Objective: We sought to determine whether an acupuncture technique specially developed for a surgical oncology population (intervention) reduces pain or analgesic use after thoracotomy compared with a sham acupuncture technique (control).

\begin{abstract}
Methods: One hundred sixty-two patients with cancer undergoing thoracotomy were randomized to group A (preoperative implantation of small intradermal needles that were retained for 4 weeks) or group B (preoperative placement of sham needles at the same schedule). The numeric rating scale of pain and total opioid use was evaluated during the in-patient stay, and the Brief Pain Inventory and Medication Quantification Scale were evaluated after discharge up to 3 months after the operation.
\end{abstract}
Results: The principal analysis, a comparison of Brief Pain Inventory pain intensity scores at the 30-day follow- up, showed no significant difference between the intervention and control groups. Pain scores were marginally higher in the intervention group $(0.05 ; 95 \%$ confidence interval, 0.74 to $-0.64 ; P=.9)$. There were also no sta- tistically significant differences between groups for secondary end points, including chronic pain assessments at 60 and 90 days, in-patient pain, and medication use in the hospital and after discharge.

Conclusion: A special acupuncture technique, as provided in this study, did not reduce pain or use of pain medication after thoracotomy more than a sham technique.

Earn CME credits at

http://cme.ctsnetjournals.org

Thoracotomy is associated with high levels of pain in the immediate postoperative period (acute postthoracotomy pain). Some patients experience continuing pain lasting greater than 60 days, termed postthoracotomy pain syndrome (chronic postthoracotomy pain). This pain can persist for years. In one study about $30 \%$ of patients still experience pain up to 4 to 5 years after the operation. ${ }^{1}$ Trauma to the intercostal nerve is the most likely cause. The pain has neuropathic and nonneuropathic components. Postthoracotomy pain causes distress and impediment of pulmonary function and mobility, leading to increased postoperative morbidity. Acute pain is managed with 1 or more of a number of strategies, including patient-controlled analgesia and epidural,

From the Integrative Medicine Service, Department of Medicine, ${ }^{a}$ the Thoracic Service, Department of Surgery, ${ }^{b}$ the Department of Epidemiology and Biostatistics, ${ }^{c}$ and the Department of Anesthesiology, ${ }^{\mathrm{d}}$ Memorial Sloan-Kettering Cancer Center, New York, New York.

The study was supported by National Cancer for Complementary and Alternative Medicine grant AT002989.

Received for publication Feb 21, 2008; revisions received June 12, 2008; accepted for publication July 26, 2008.

Address for reprints: Gary Deng, MD, PhD, Integrative Medicine Service, Department of Medicine, Memorial Sloan-Kettering Cancer Center, 1429 First Avenue, New

York, NY 10021 (E-mail: dengg@mskcc.org).

J Thorac Cardiovasc Surg 2008;136:1464-9

$0022-5223 / \$ 34.00$

Copyright (c) 2008 by The American Association for Thoracic Surgery

doi: 10.1016/j.jtcvs.2008.07.053 paravertebral, and other interventions. The treatment of chronic postthoracotomy pain is more difficult. ${ }^{1-3}$

Acupuncture is a complementary medicine modality that originated in traditional Chinese medicine practices. ${ }^{4}$ Clinical studies have documented its benefit in the management of certain painful conditions, such as joint osteoarthritis, ${ }^{5}$ pain during labor, ${ }^{6}$ lower back pain, ${ }^{7}$ pain after oral surgery, ${ }^{8}$ and pain after abdominal surgery. ${ }^{9}$ The study by Kotani and colleagues ${ }^{9}$ is especially relevant to postthoracotomy pain. In this randomized controlled study a special acupuncture technique, insertion of intradermal needles preoperatively, significantly reduced pain scores in the first 2 days after the operation when compared with a sham technique. This technique could be more applicable in Western medicine practice than traditional acupuncture because patients can be sent home with indwelling needles, obviating the need for repeated hospital visits to receive acupuncture treatment.

Inspired by the study of Kotani and colleagues, ${ }^{9}$ we conducted and published a pilot study to demonstrate the feasibility of using intradermal acupuncture needles to treat postthoracotomy pain. ${ }^{10}$ Here we report data from a subsequent randomized controlled trial to determine whether placement of intradermal acupuncture needles is superior to the sham technique in the treatment of acute and chronic postthoracotomy pain.

\section{MATERIALS AND METHODS \\ Study Design}

This was a randomized, sham-controlled, subject-blinded trial. The duration of the intervention was 4 weeks. 


\section{Abbreviations and Acronyms \\ BPI = Brief Pain Inventory \\ $\mathrm{CI}=$ confidence interval \\ MQS = Medication Quantification Scale \\ MSKCC $=$ Memorial Sloan-Kettering Cancer Center}

\section{Study Subjects}

Informed consent was obtained before subject enrollment according to a clinical trial protocol approved by the Institutional Review Board at Memorial Sloan-Kettering Cancer Center (MSKCC). Recruitment took place between February 2005 and January 2007.

All study participants were patients with cancer aged 18 years or older scheduled for unilateral thoracotomy at MSKCC. Patients meeting these criteria were excluded if their operations involved chest wall involvement, esophagectomy, or the hemiclamshell, clamshell, or extrapleural approaches because these more extensive procedures have a higher risk of complications. Patients who had received acupuncture treatment in the previous 6 weeks also were ineligible to discount any persisting effect of acupuncture. Additional ineligibility factors included patients with platelet counts of less than 20,000, an international normalized ratio of greater than 2.5 , or an absolute neutrophil count of less than 0.5 ; known cardiac conditions with high or moderate risk of endocarditis, as defined by the American Heart Association, ${ }^{11}$ because the intradermal acupuncture needles remained in the skin for up to 4 weeks; inability to remove the needles without assistance; or no home assistance and were unable or unwilling to return to the hospital if they elected to remove the needles before their postdischarge visit.

\section{Randomization}

Randomization was stratified by epidural anesthesia (yes/no) using blocks of random length and accomplished by using a secure, password-protected institutional computer system stratified by permuted blocks of random length. The system is designed to ensure that allocation cannot be guessed before a patient is registered or changed afterward, thus ensuring full allocation concealment. After subject registration and randomization, a research assistant who was otherwise unconnected with the trial accessed allocation and telephoned the acupuncturist with details of allocation. Patients were blind to study group; the acupuncturists and the designated research assistant were aware of which patients received true treatment and which received sham treatment.

\section{Intervention}

Within 2 hours before the operation and after placement of an epidural catheter but before induction of anesthesia, 9 small intradermal acupuncture needles were inserted in a sterile fashion on each side of the spine corresponding to the BL-12 to BL-19 acupuncture points and an extra point (Wei Guan Xia Shu, covering the T2 to T9 dermatomes). The BL points are points comparable with those demonstrated to have antinociceptive effects in prior trials of abdominal surgery (T9-L3 dermatomes) ${ }^{9}$ but located more rostrally. In addition, 1 stud was placed in each leg (ST-36 point) and 1 in each auricle (Shenmen point). ST-36 and Shenmen are commonly used to treat pain.

The sterile intradermal needles, which we term studs, are approximately $1.5 \mathrm{~mm}$ long, with a diameter of approximately $0.2 \mathrm{~mm}$. They have the appearance of miniature thumbtacks. On placement, the short needle penetrates the epidermis and provides stimulation, whereas the ring at the end of the stud (4 mm in diameter) keeps it from entering the skin completely and being lost in the subcutaneous tissue (Figure 1). The needles are shorter than what was used in the study by Kotani and colleagues ${ }^{9}(1.5 \mathrm{vs} 5 \mathrm{~mm}$ ). The studs were covered with Tegaderm transparent films (3M, St Paul, Minn), which are impermeable to microorganisms but permeable to both water

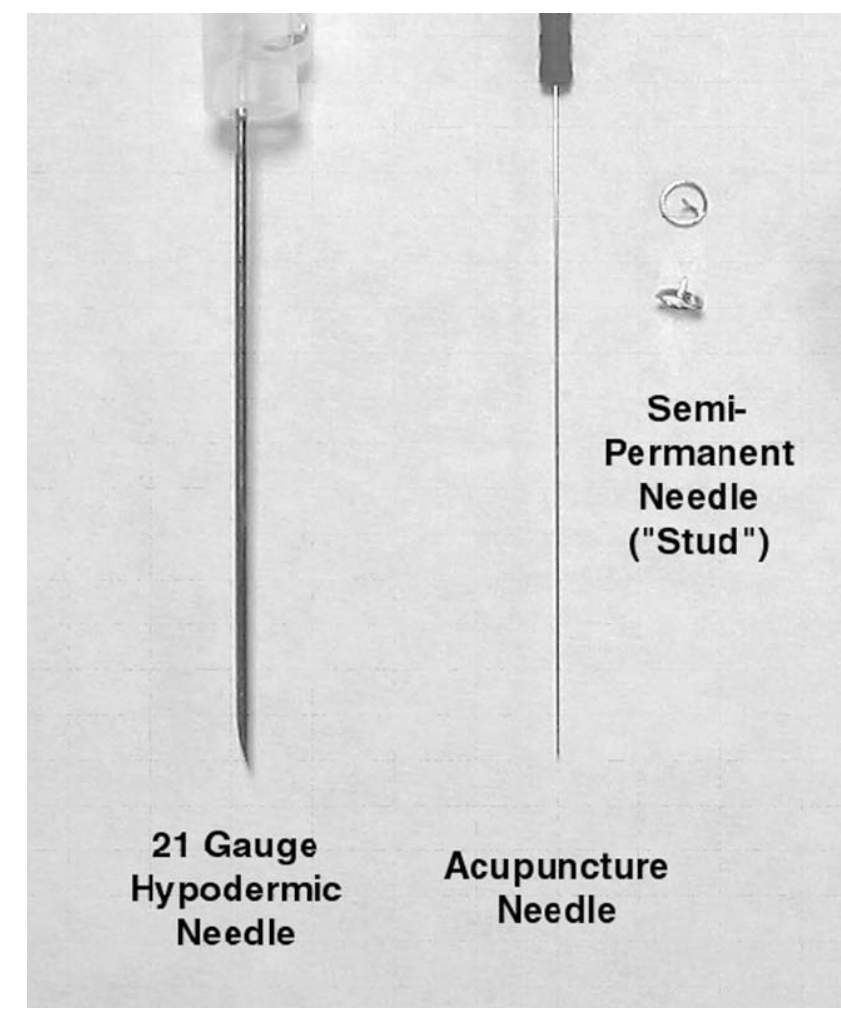

FIGURE 1. Acupuncture needles.

vapor and air. Preoperative acupuncture took place in the MSKCC Presurgical Center. The studs were exchanged with new ones 1 week after the initial placement. Those at ST-36 and Shenmen were removed 1 week later. Those at the back were removed 3 weeks later. The total period of acupuncture intervention was 4 weeks. We believed that a long period of stimulation would be required given the severity of thoracotomy pain.

Treatment in the control group was identical to that for the true acupuncture group, with the following exceptions. Studs placed in the back were dummy studs with a ring but no needle. The back studs were placed halfway between the upper and lower border of the spinous processes T2 to T10 approximately 0.5 cun (approximately $1.25 \mathrm{~cm}$ ) from the spine. The leg studs were placed at 2 cun (approximately $5 \mathrm{~cm}$ ) posterior to GB-34 on the posterior of the lower leg. No studs were placed in the ear. Instead, sham studs were placed on the anterior arm, 3 cun (approximately $5 \mathrm{~cm}$ ) proximal and 3 cun (approximately $5 \mathrm{~cm}$ ) medial to the midpoint of the antecubital crease. There are no known acupuncture points near these 2 locations. Sham studs were placed at different points to prevent tactile stimulation at acupuncture points.

\section{Evaluation}

The primary end point was the Brief Pain Inventory (BPI) pain intensity score at day 30 after the operation. The BPI is a validated pain measurement tool that measures both the intensity of pain (sensory dimension) and the interference of pain in the patient's life (reactive dimension). ${ }^{12}$ On the BPI, mild pain is defined as a worst pain score of 1 to 4 , moderate pain is defined as a worst pain score of 5 to 6 , and severe pain is defined as a worst pain score of 7 to 10 . Secondary end points included BPI, a 0 - to 10-point numeric rating scale of pain, total opioid use, and Medication Quantification Scale (MQS) scores at 1, 2, and 4 weeks (for acute postthoracotomy pain) and at 2 and 3 months postoperatively (for chronic postthoracotomy pain). The MQS is a reliable, valid, and sensitive method of assessing medication use in patients with chronic pain in whom drugs are scaled relative to their recommended daily doses. ${ }^{13}$ 


\section{Statistical Methods}

Sample size calculations were based on data from our pilot study. ${ }^{10}$ The mean BPI pain score at the 30-day follow-up in the pilot study was 2.7, with a standard deviation of 1.48. As a conservative measure, we took the upper 75th percentile of the standard deviation (1.63). Although our analysis was adjusted for baseline score, we did not incorporate any further correction to the standard deviation on the assumption of a low correlation between baseline and follow-up scores. We set a minimum clinically significant difference of $25 \%$, resulting in a hypothesized score in the control group close to 3.6. With a $5 \% \alpha$ value and a power of $80 \%, 52$ evaluable patients per group were required.

The principal analysis was a comparison of BPI pain intensity scores at the 30-day follow-up. Secondary analyses were BPI total and pain interference at 30 days; BPI scores after discharge at 60 and 90 days; areas under the curve for numeric rating scale pain at rest, on movement, and on coughing for the first 5 days after the operation; total opiate use in milligrams of oral morphine equivalents; and nonopiate MQS scores in the first 5 days after the operation. All analyses were conducted by using analysis of covariance, with the randomization stratum (epidural anesthesia) as the covariate. In the BPI analyses baseline BPI scores were used as a covariate. Baseline BPI scores were missing for 15 patients. In patients who did provide baseline BPI data, scores were low and were not strongly correlated with other variables. Accordingly, we used simple mean imputation to obtain a score to enter into the model. Patients were analyzed in their randomized groups, regardless of treatment assignment. There were, however, 2 exceptions to the intention-to-treat principle: patients who did not receive operations or those who were judged by a blinded investigator to have had no chance of receiving acupuncture were excluded from analysis. An example of the latter would be a patient whose operation was moved 2 hours forward because of a change in scheduling, such that the acupuncturist arrived after the patient had been sent to the operating room. Sensitivity analysis was planned to account for missing posttreatment data, but this was found not to be warranted. All analyses were conducted with Stata 9.2 software (StataCorp, College Station, Tex).
TABLE 1. Baseline characteristics of study subjects who provided data for the primary end point

\begin{tabular}{lcc}
\hline \multicolumn{1}{c}{ Allocation } & Acupuncture & Sham \\
\hline No. & 52 & 54 \\
Age (y), median & $65(58-72)$ & $63(57-70)$ \\
$\quad$ (interquartile range) & & \\
Female sex & $24(46 \%)$ & $30(56 \%)$ \\
Epidural & $42(81 \%)$ & $43(80 \%)$ \\
Operation & & \\
$\quad$ Full & $3(6 \%)$ & $6(11 \%)$ \\
$\quad$ Partial & $49(94 \%)$ & $48(89 \%)$ \\
\hline
\end{tabular}

\section{RESULTS}

Participant flow through the trial is shown in Figure 2. Table 1 shows the baseline characteristics of the study subjects. The acupuncture and sham groups are well balanced for age, sex, use of epidural analgesia, and operation type. About $80 \%$ of the participants received epidural analgesia. The majority of patients $(118,72 \%)$ were accrued by 3 of 6 surgeons, with all surgeons accruing equal numbers of patients to each arm of the study.

Pain scores during follow-up, as measured by using the BPI at days 10 and 30 (for "acute postthoracotomy pain"), as well as days 60 and 90 (for "chronic postthoracotomy pain"), are shown in Table 2 . In the primary analysis (day 30) pain scores were marginally higher in the acupuncture group $(0.05 ; 95 \%$ confidence interval $[\mathrm{CI}], 0.74$ to $-0.64 ; P=.9)$. There were no significant differences between groups for any BPI domains at any follow-up.

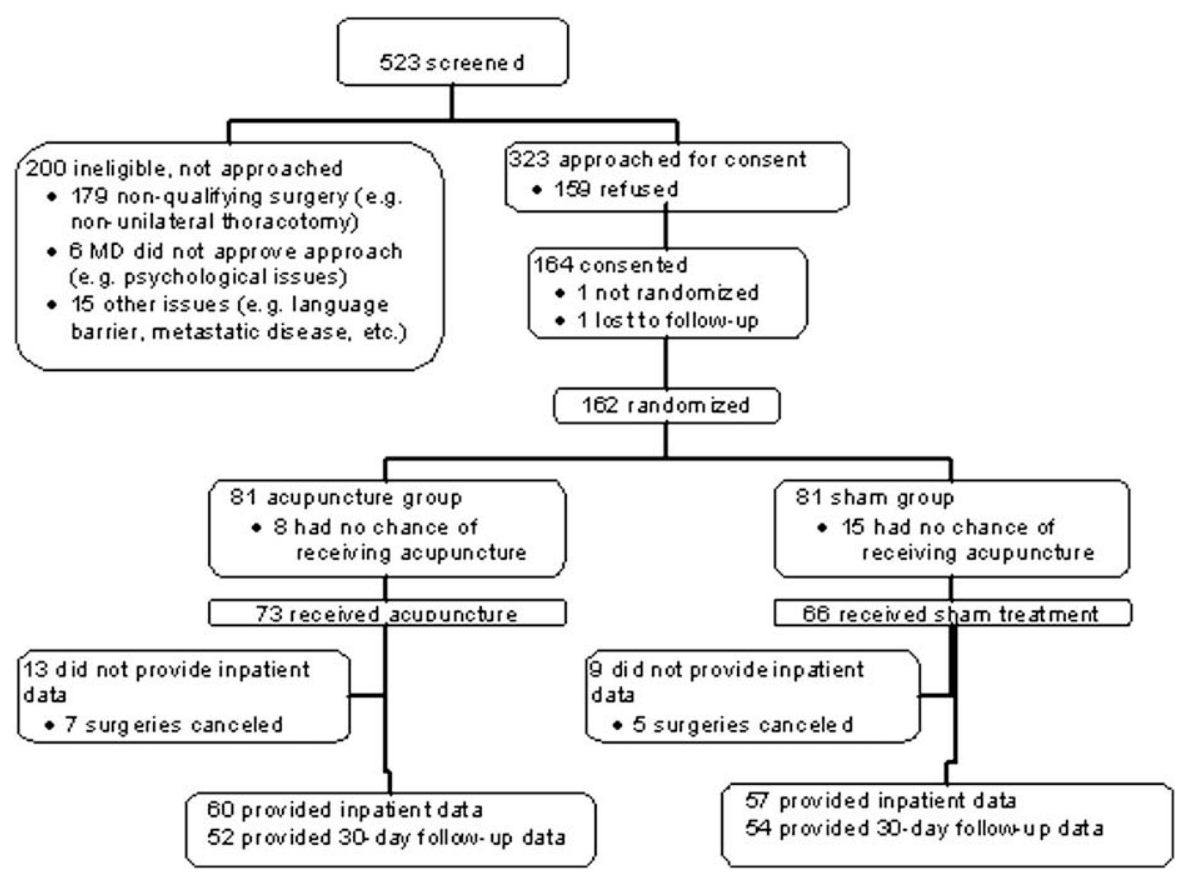

FIGURE 2. Participant flow (description and number of subjects). 
TABLE 3. Numeric rating scale scores during the inpatient stay

\begin{tabular}{lcccc}
\hline \multicolumn{1}{c}{ Variable } & $\begin{array}{c}\text { Acupuncture } \\
(\mathbf{n = 6 2})\end{array}$ & $\begin{array}{c}\text { Sham } \\
(\mathbf{n}=\mathbf{5 7})\end{array}$ & $\begin{array}{c}\boldsymbol{P} \\
\text { Difference* }\end{array}$ & $\begin{array}{c}\text { value } \\
\text { Pain at rest }\end{array}$ \\
& $2.53(1.86)$ & $2.96(2.00)$ & $\begin{array}{c}0.44(-0.26 \text { to } \\
1.13)\end{array}$ & .2 \\
Pain on movement & $4.53(2.10)$ & $4.40(2.03)$ & $\begin{array}{c}-0.13(-0.86 \text { to } \\
0.61)\end{array}$ & .7 \\
& & & $0.05(-0.80$ to & .9 \\
Pain while coughing & $5.71(2.37)$ & $5.76(2.41)$ & $0.89)$ & \\
& & & \\
\hline
\end{tabular}

Data are presented as the mean (standard deviation). *Difference between groups positive value indicates lower pain in acupuncture group. Values in parentheses are the $95 \%$ confidence interval.

Moreover, the $95 \%$ CIs rarely include a difference in favor of acupuncture of greater than 1 point. Pain scores during hospitalization, as recorded by using numeric rating scales, are shown in Table 3. Again, there are no statistically significant differences between groups. Figure 3 shows the acute pain score, averaged for the 3 domains, separately for the acupuncture and sham groups.

Whether the patients received an epidural or not did not appear to affect the results. All analyses were conducted by using analysis of covariance with the randomization stratum (epidural anesthesia) as the covariate, meaning that the analysis was done for those receiving an epidural, for those not receiving an epidural, and for the combined population. There were no significant differences in the results among these 3 subgroups.

Nineteen $(32 \%)$ patients in the control group received antiemetics compared with $23(37 \%)$ patients in the acupuncture group (odds ratio for acupuncture, $1.25 ; 95 \% \mathrm{CI}, 0.59$ 2.65; $P=.6$ ). Pain medication use is described in Table 4 . Medication use was similar in each group. Blinding was adequate: of patients who expressed a belief about assignment in response to questions about blinding, 14 of 26 in the acupuncture group and 16 of 27 in the sham group guessed correctly.

\section{DISCUSSION}

Postthoracotomy pain is a common problem after thoracic surgery. In some patients the pain persists for months or even years after the operation. In addition to pharmacologic and behavioral therapies, acupuncture offers a potential therapeutic option, given previous reports of its benefit in treating other types of postoperative pain. However, traditional acupuncture techniques require multiple treatments by the acupuncturist, which might hinder its acceptance in Western clinical practice. In this study we evaluated a special acupuncture technique, perioperative stimulation with implanted small intradermal acupuncture needles, which requires only 2 encounters with the acupuncturist. A similar technique was previously reported to reduce pain after abdominal surgery. However, in this study we found that this particular acupuncture technique did not significantly reduce acute or chronic postthoracotomy pain nor did it 


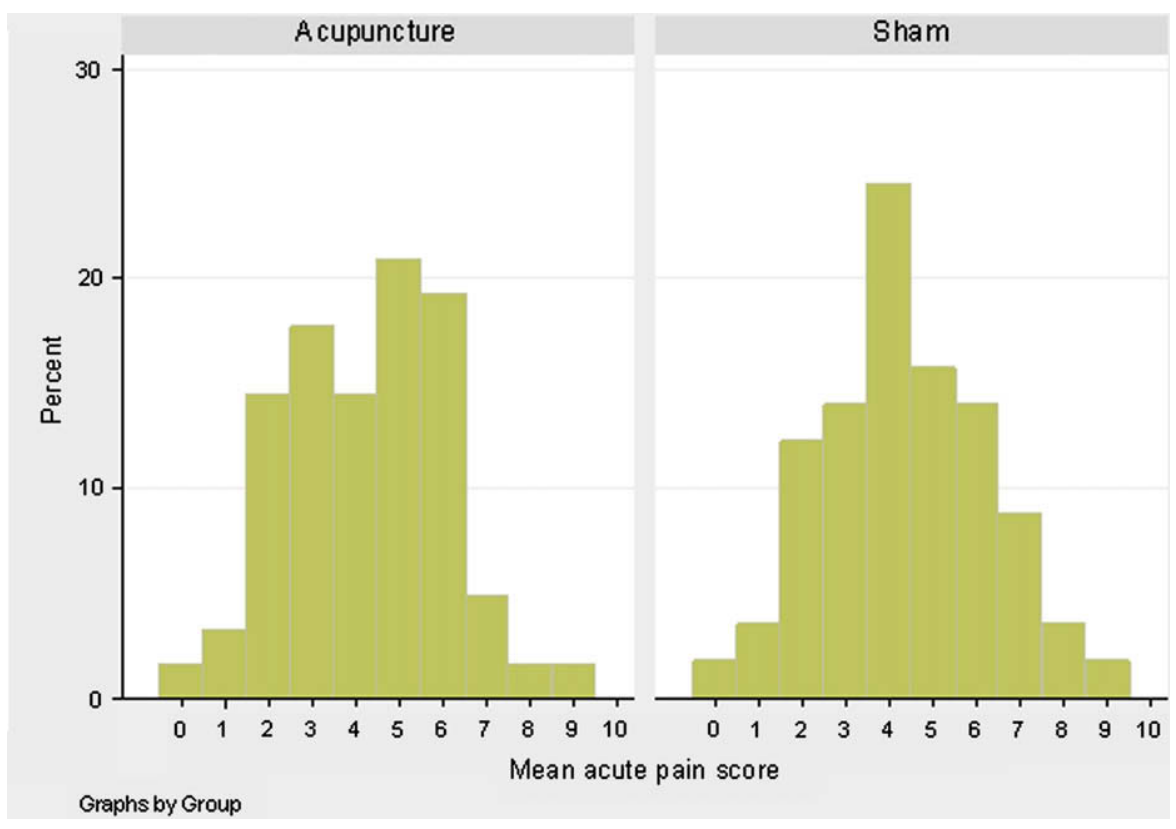

FIGURE 3. Histogram of acute pain scores by group.

reduce the use of pain medication when compared with a sham technique.

There could be several explanations for this result. First, acupuncture might be ineffective for postthoracotomy pain. Yet several previous controlled studies showed that acupuncture provided analgesia after abdominal ${ }^{14,15}$ and thoracic $^{16}$ surgery. A second possible explanation is that the acupuncture technique used in this study might not deliver strong enough stimulation to produce analgesic effects.

A recently published, randomized, sham-controlled study of acupuncture did show reduction of pain medicine requirements in patients after thoracotomy. In that study electrical stimulation was applied to acupuncture needles, and treatment was given twice daily for 7 postoperative days. When compared with sham acupuncture, there was no statistically significant difference in pain scores between the 2 groups, but the cumulative dose of patient-controlled analgesia (morphine) used on postoperative day 2 was significantly lower in the electroacupuncture group. ${ }^{16}$ Our intervention regimen provided less intense stimulation (implanted intradermal needles vs electroacupuncture) but over a longer period of time (4 weeks vs 1 week).

TABLE 4. Medication scores

\begin{tabular}{lcccc}
\hline \multicolumn{1}{c}{ Variable } & $\begin{array}{c}\text { Acupuncture } \\
(\mathbf{n}=\mathbf{6 3})\end{array}$ & $\begin{array}{c}\text { Sham } \\
(\mathbf{n = 5 9 )}\end{array}$ & Difference* $^{*}$ & $\begin{array}{c}\boldsymbol{P} \\
\text { value }\end{array}$ \\
\hline Medication & 10.37 & 11.14 & $0.76(-1.88$ & .6 \\
$\quad$ quantification scale & $(6.96)$ & $(7.78)$ & to 3.40) & \\
Morphine & 1530 & 1563 & $28(-330$ & .9 \\
$\quad$ equivalents (mg) & $(1172)$ & $(1093)$ & to 386) & \\
\hline
\end{tabular}

Data are presented as the mean (standard deviation). *Difference between groups: positive value indicates lower pain in acupuncture group. Values in parentheses are the $95 \%$ confidence interval.
Our rationale for selecting this technique was based on 2 factors. First, a previous randomized controlled trial reported that preoperative intradermal acupuncture studs reduced postoperative pain, nausea and vomiting, analgesic requirement, and sympathoadrenal responses. ${ }^{9}$ This technique was thought to deliver less intense but longer-lasting stimulation, possibly more effective in the treatment of persisting postoperative pain. ${ }^{9}$ Second, traditional acupuncture regimens require frequent treatments by acupuncturists. In the current Western medical practice setting, this presents an access barrier because many patients do not have the time or financial resources to return often for treatment. If the stud technique applied in this study was efficacious, we believe that it would find a wider application because it requires only preoperative placement of acupuncture needle and 1 postoperative visit to replace the needles.

Because in this study perioperative stimulation with intradermal acupuncture studs failed to show significant reduction in pain or in use of postoperative analgesic medication, we conclude that the acupuncture technique as provided in this study cannot be recommended for the prevention or treatment of postthoracotomy pain. The results of our study might not be generalizable to other acupuncture techniques or to other types of pain. Further research should apply a more intensive acupuncture regimen similar to those reported previously. ${ }^{16}$

We appreciate the contributions of Drs Kenneth Cubert and Neil Patel from the Department of Anesthesiology and thank Jennifer Lee, Cheryl Co, Erika Greenidge, Kristine Brown, and Elizabeth Seidler (Research Study Assistants) and Yi Chan, Chunyan Teng, K. Simon Yeung, Yi Lily Zhang, Carole Johnson, Sally Kao, and Sissi Le (acupuncturists) for their work. 


\section{References}

1. Karmakar MK, Ho AM. Postthoracotomy pain syndrome. Thorac Surg Clin. 2004; 14:345-52.

2. Katz J, Jackson M, Kavanagh BP, Sandler AN. Acute pain after thoracic surgery predicts long-term post-thoracotomy pain. Clin J Pain. 1996;12:50-5.

3. Koehler RP, Keenan RJ. Management of postthoracotomy pain: acute and chronic. Thorac Surg Clin. 2006;16:287-97.

4. Kaptchuk TJ. Acupuncture: theory, efficacy, and practice. Ann Intern Med. 2002; 136:374-83.

5. Kwon YD, Pittler MH, Ernst E. Acupuncture for peripheral joint osteoarthritis: a systematic review and meta-analysis. Rheumatology (Oxford). 2006;45:1331-7.

6. Smith CA, Collins CT, Cyna AM, Crowther CA. Complementary and alternative therapies for pain management in labour. Cochrane Database Syst Rev. 2006;(4): CD003521.

7. Manheimer E, White A, Berman B, Forys K, Ernst E. Meta-analysis: acupuncture for low back pain. Ann Intern Med. 2005;142:651-63.

8. Lao L, Bergman S, Hamilton GR, Langenberg P, Berman B. Evaluation of acupuncture for pain control after oral surgery: a placebo-controlled trial. Arch Otolaryngol Head Neck Surg. 1999;125:567-72.

9. Kotani N, Hashimoto H, Sato Y, Sessler DI, Yoshioka H, Kitayama M, et al. Preoperative intradermal acupuncture reduces postoperative pain, nausea and

vomiting, analgesic requirement, and sympathoadrenal responses. Anesthesiology. 2001;95:349-56.

10. Vickers AJ, Rusch VW, Malhotra VT, Downey RJ, Cassileth BR. Acupuncture is a feasible treatment for post-thoracotomy pain: results of a prospective pilot trial. BMC Anesthesiol. 2006;6:5.

11. Dajani AS, Taubert KA, Wilson W, Bolger AF, Bayer A, Ferrieri P, et al. Prevention of bacterial endocarditis. Recommendations by the American Heart Association. Circulation. 1997;96:358-66.

12. Cleeland CS, Ryan KM. Pain assessment: global use of the Brief Pain Inventory. Ann Acad Med Singapore. 1994;23:129-38.

13. Masters Steedman S, Middaugh SJ, Kee WG, Carson DS, Harden RN, Miller MC Chronic-pain medications: equivalence levels and method of quantifying usage. Clin J Pain. 1992;8:204-14.

14. Lin JG, Lo MW, Wen YR, Hsieh CL, Tsai SK, Sun WZ. The effect of high and low frequency electroacupuncture in pain after lower abdominal surgery. Pain. 2002;99:509-14.

15. Sim CK, Xu PC, Pua HL, Zhang G, Lee TL. Effects of electroacupuncture on intraoperative and postoperative analgesic requirement. Acupunct Med. 2002;20:56-65.

16. Wong RH, Lee TW, Sihoe AD, Wan IY, Ng CS, Chan SK, et al. Analgesic effect of electroacupuncture in postthoracotomy pain: a prospective randomized trial. Ann Thorac Surg. 2006;81:2031-6. 